\title{
Protein- and Sequencing-based Massively Parallel Single-cell Approaches to Gene Expression Profiling
}

Xuhuai Ji๋, Igor Goncharov, Miao Zhao, Molly Miranda and Holden T. Maecker

\author{
Human Immune Monitoring Center (HIMC), Institute for Immunity, Transplantation and Infection, \\ Department of Microbiology \& Immunology, Stanford University, Palo Alto, CA 94305, USA \\ *For correspondence: xuhuai.ji@stanford.edu
}

\begin{abstract}
[Abstract] Understanding cellular diversity and dynamics in immune cells are critical for elucidating mechanisms of diagnosis, response to therapeutics, and patient prognosis. Our goal is to apply a technically simple approach for gene expression cytometry combining next-generation sequencing with stochastic barcoding of single cells. The BD Rhapsody Single-cell Analysis System was implemented to assess cellular heterogeneity present in 10 Dynabead-sorted human $\mathrm{T}$ cell samples. We captured and sequenced over 40,000 single cells across 10 samples. We used oligonucleotide-conjugated antibodies and an immune targeted gene panel that utilizes multiplex PCR for detection of several hundred genes of interest. A combinatorial library of beads bearing cell- and molecular-barcoding capture probes was used to uniquely label transcripts. This allowed for reconstruction of the digital gene expression profile of thousands of individual cells in a single experiment without the need for robotics or automation. We demonstrate the ability of the BD Rhapsody targeted panel to distinguish six different cell types and 18 distinct gene-expression biomarkers, including CLEC4E, CSF2, IL1RN, TPSAB1, CCL19, CCL22, CXCL2, LAMP3, SPP1, and VMO. The BD Rhapsody targeted panel yields more sequencing depth with much fewer $(<2 \%)$ sequencing reads per cell. The measurement of specific proteins and transcripts in individual cells is critical for understanding the role of cellular diversity in development, health, and disease.
\end{abstract}

Keywords: AbSeq, Gene Expression, Targeted RNAseq, Rhapsody, Single Cell RNAseq, Molecular Indexing

[Background] Analyzing individual blood cells can identify their specific role in fighting disease. To understand why cells differ from each other, we need to understand which genes are transcribed (and ultimately expressed as proteins) at a single-cell level. Understanding cellular diversity at both protein and transcriptional levels is critical for elucidating mechanisms of pathogenesis, response to therapeutics, and disease prevention (Han et al., 2014; Landhuis, 2018). The BD Rhapsody Single-cell Analysis System is designed to assess cellular heterogeneity present in biological samples by capturing and sequencing thousands of single cells. The system uses oligonucleotide-conjugated antibodies and a targeted gene panel that utilizes multiplex PCR for detecting hundreds of genes of interest in immunity or cancer (Newell et al., 2012; Wills et al., 2013). A combinatorial library of beads bearing cell- and molecular-barcoding capture probes is used to uniquely label transcripts (UMI). 
This allows for reconstruction of the digital gene expression profile of thousands of individual cells in a single experiment without the need for robotics or automation (Shapiro et al., 2013; Fu et al., 2014a and 2014b). Rather than probing the whole transcriptome, this technology focuses on several hundred transcripts, which cuts the number of sequencing reads about tenfold. The BD Rhapsody Targeted Panel can yield more sequencing depth with much fewer $(<2 \%)$ sequencing reads per cell and thus facilitates a more affordable single-cell snapshot of heterogeneous subpopulations in single live cells (Fan et al., 2015; Lam et al., 2018; Martin et al., 2018). High sensitivity is demonstrated by the detection of lowabundance transcripts and rare cells. We implemented the Rhapsody Single-cell Analysis System (Reference 1) to assess cellular heterogeneity present in PBMC in this study. BD Rhapsody empowers single-cell data by making experiments easier and more cost-efficient, without using robotics or automation. There are basically no limitations for this approach, as long as there are enough cells (> 1,000 cells) to start with. No other alternative methods for such targeted RNAseq + Abseq are commercially available at the moment.

\section{Materials and Reagents}

1. Gilson ${ }^{\mathrm{TM}}$ PIPETMAN ${ }^{\mathrm{TM}}$ Tipack $^{\mathrm{TM}}$ filter tips, $100-1,200 \mu$ l for BD Rhapsody ${ }^{\mathrm{TM}} \mathrm{P} 1200 \mathrm{M}$ pipette (Thermo Fisher Scientific, catalog number: F171803G)

2. Gilson $^{\mathrm{TM}}$ PIPETMAN ${ }^{\mathrm{TM}}$ Tipack $^{\mathrm{TM}}$ Racked pipet tips, $500-5,000 \mu$ for BD Rhapsody ${ }^{\mathrm{TM}} \mathrm{P} 5000 \mathrm{M}$ pipette (Thermo Fisher Scientific, catalog number: F161370G)

3. Falcon ${ }^{\circledR}$ tube with cell strainer cap (Thermo Fisher Scientific, catalog number: 352235)

4. DNA LoBind tubes, $1.5 \mathrm{ml}$ (Eppendorf, catalog number: 0030108051)

5. DNA LoBind tubes, $5 \mathrm{ml}$ (Eppendorf, catalog number: 0030108310)

6. Low retention filtered pipette tips, $10 \mu \mathrm{l}$ (Gilson PIPETMAN, catalog number: 1222Q28; RAININ, catalog number: 17015169)

7. Low retention filtered pipette tips, $200 \mu \mathrm{l}$ (Gilson PIPETMAN, catalog number: 1222Q31; RAININ, catalog number:17014963)

8. Low retention filtered pipette tips, 1,000 $\mu$ (Gilson PIPETMAN, catalog number: 1222Q34; RAININ, catalog number: 17015122)

9. Qubit ${ }^{\mathrm{TM}}$ assay tubes (Thermo Fisher Scientific, catalog number: Q32856)

10. $0.2 \mathrm{ml} \mathrm{PCR} \mathrm{12-strip} \mathrm{tubes} \mathrm{(Axygen,} \mathrm{catalog} \mathrm{number:} \mathrm{14-222-259)}$

11. $10 \mathrm{ml}$ sterile serological pipettes (Fisher Scientific, catalog number: 13-676-10F)

12. $15 \mathrm{ml}$ tubes (Conical Centrifuge Tubes, Fisher Scientific, catalog number: 14-959-53A)

13. Waste collection container (BD Biosciences, PN 650000090)

14. Agencourt ${ }^{\circledR}$ AMPure ${ }^{\circledR}$ XP magnetic beads (Beckman Coulter, catalog number: A63880)

15. BD ${ }^{T M}$ Stain Buffer (FBS) (BD Biosciences, catalog number: 554656)

16. Calcein AM cell permeant dye (Thermo Fisher Scientific, catalog number: $C 1430$ )

17. DRAQ7 ${ }^{\mathrm{TM}}, 0.3 \mathrm{mM}$ (BD Biosciences, catalog number: 564904$)$

18. Dimethyl sulfoxide (DMSO) (Corning, catalog number: $25950 \mathrm{CQC}$ ) 
19. $70 \%$ ethyl alcohol or $70 \%$ isopropyl alcohol

20. Ethyl alcohol, Pure (200 proof, molecular biology grade) (Sigma-Aldrich, catalog number: E7023-500ML)

21. 1x RBC Lysis Buffer (Thermo Fisher Scientific, catalog number: 00-4333-57)

22. Phosphate-buffered saline (calcium- and magnesium-free) (Gibco, catalog number: 10-010-023)

23. Premoistened cleaning wipes with $70 \%$ ethyl alcohol or $70 \%$ isopropyl alcohol

24. BD Rhapsody Cartridge Reagent Kit (BD Genomics, PN 633731)

25. BD Rhapsody Cartridge Kit (BD Genomics, PN 633733)

26. BD Rhapsody cDNA Kit (BD Genomics, PN 633732)

27. BD Rhapsody Targeted Amplification Kit (BD Genomics, PN 633734)

28. BD Single-cell Multiplexing Kit-Human Sample Tag (12) Component (BD Genomics)

29. BD Single-cell Multiplexing Kit Library Amplification Component (BD Genomics, PN 633782)

30. BD Rhapsody ${ }^{\mathrm{TM}}$ Immune Response Panel Hs (BD Genomics, PN 633750)

31. Qubit ${ }^{\top \mathrm{M}}$ dsDNA HS Assay Kit (Thermo Fisher Scientific, catalog number: Q32851)

32. Agilent Bioanalyzer DNA High Sensitivity Kit (Agilent Technologies, catalog number: 5067-4626)

33. $10 \%$ bleach (Thermo Fisher Scientific, catalog number: 50-163-3379)

34. cDNA master mix prep (see Recipes)

35. Exonuclease I master mix (see Recipes)

36. PCR1 reaction master mix (see Recipes)

37. PCR Program 1 (see Recipes)

38. Targeted PCR2 reaction master mix (see Recipes)

39. Sample Tag PCR2 reaction master mix (see Recipes)

40. Targeted final amplification master mix (see Recipes)

41. Sample Tag final amplification master mix (see Recipes)

42. PCR Program 2 (see Recipes)

\section{Equipment}

1. Pipettes (P2, P10, P20, P200, P1000) (RAININ Single channel Pipet-Lite XLS, P2: catalog number:17014393; P10: catalog number: 17014388; P20: catalog number: 17014392; P200: catalog number: 17014391; P1000: catalog number: 17014382)

2. Multi-channel pipette, $\mathrm{P} 2-20 \mu \mathrm{l}$ or $\mathrm{P} 20-200 \mu \mathrm{l}$ (RAININ Multichannel Pipet-Lite XLS, P2-20: catalog number: 17013808; P20-200: catalog number: 17013810)

3. Pipet-Aid (Drummond, catalog number: 13-681-06)

4. Illumina HiSeq 4000 sequencer (Illumina, HiSeq 4000 system)

5. Laminar flow hood (AirClean System AC600, catalog number: 36-100-4377)

6. Digital timer (Fisherbrand, catalog number: 06-662-5)

7. Vortexer (Fisherbrand, catalog number: 02-215-414) 
8. 6-Tube Magnetic Separation Rack for $1.5 \mathrm{ml}$ tubes (New England Biolabs, catalog number: S1506S)

9. Large magnetic separation stand (V\&P Scientific, catalog number: VP 772FB-1)

10. Clear acrylic cylinder adapter for $15 \mathrm{ml}$ tube magnet (V\&P Scientific, catalog number: VP 772FB1A)

11. Low-profile magnetic separation stand for $0.2 \mathrm{ml}$, 8-strip tubes (V\&P Scientific, or Clontech, catalog numbers: VP $772 \mathrm{~F} 4-1$ or 635011$)$

12. Improved Neubauer Hemocytometer (INCYTO, model: DHC-N01-5)

13. BD Rhapsody Scanner (BD Genomics, PN 633701)

14. Sample Loading Station (BD Genomics, PN 63370)

15. ThermoMixer ${ }^{\circledR} \mathrm{C}$ (Eppendorf, catalog number: 5382000023)

16. SmartBlock ${ }^{\mathrm{TM}}$ Thermoblock $1.5 \mathrm{ml}$ (Eppendorf, catalog number: 5360000038 )

17. Qubit ${ }^{\mathrm{TM}} 3.0$ Fluorometer (Thermo Fisher Scientific, catalog number: Q33216)

18. $V_{W R}{ }^{\circledR}$ Advanced Mini Dry Block Heater with Heated Lid (VWR, catalog number: 10153-348)

19. 2100 Bioanalyzer (Agilent Technologies, catalog number: G2940CA)

20. Thermal cycler with heated lid (Thermo Fisher Scientific SimplAmp, catalog number: A24811)

21. Water bath or incubator at $37^{\circ} \mathrm{C}$ (Thermo Scientific, catalog number: TSCOL19)

22. Microcentrifuge for $1.5-2.0 \mathrm{ml}$ tubes (Thermo Scientific, catalog number: 75004061 )

23. Microcentrifuge for $0.2 \mathrm{ml}$ tubes (Southwest Science, catalog number: SC1006-MBlue)

24. Centrifuge and rotor for $15 \mathrm{ml}$ tubes (Eppendorf 5417C, catalog number: EP-5417C; Rotor, catalog number: A-4-44)

25. Barcode reader with keyboard wedge, USB 2.0 or 3.0 compatible (Brady Code Reader 3600 , catalog number: 19-104-473)

\section{Software}

1. BD Rhapsody ${ }^{\top M}$ Analysis pipeline (on Seven Bridges Genomics platform)

2. $B D^{\mathrm{TM}}$ Data View (run under MATLAB)

3. MATLAB Runtime (R2017b, 9.3, for Windows) (https://www.mathworks.com/products/compiler/matlab-runtime.html) 


\section{Procedure}

The workflow of the general protocol is indicated in Figure 1.

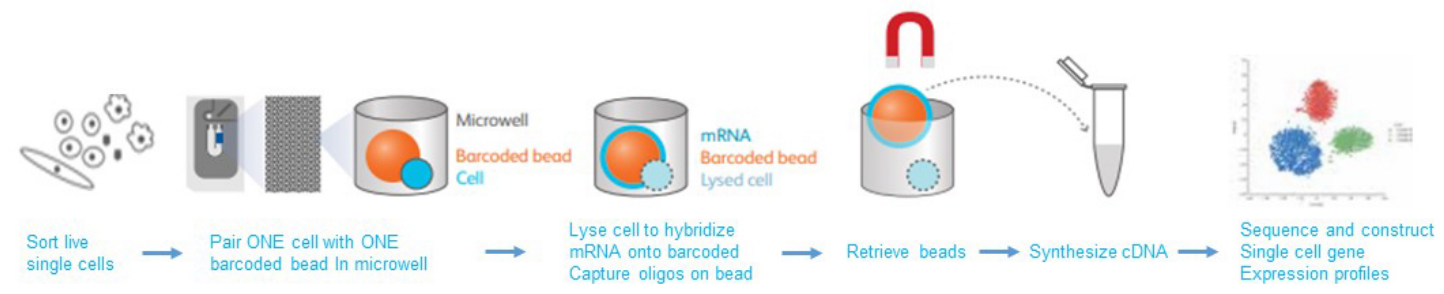

Figure 1. Scheme of this protocol workflow

A. Priming the BD Rhapsody Cartridge

1. To ensure an air-tight seal with the BD Rhapsody ${ }^{\top M}$ P1200M or P5000M pipette, hold the pipette with one hand, and slightly twist the pipette to firmly seat a pipette tip on the pipette shaft.

2. Avoid introducing bubbles while pipetting into the BD Rhapsody Cartridge.

3. Move the left slider to the middle (0) position on the BD Rhapsody ${ }^{\top M}$ Sample Loading Station. The Retrieval (top) magnet and Lysis (bottom) magnets are away from the cartridge tray (Figure 2).

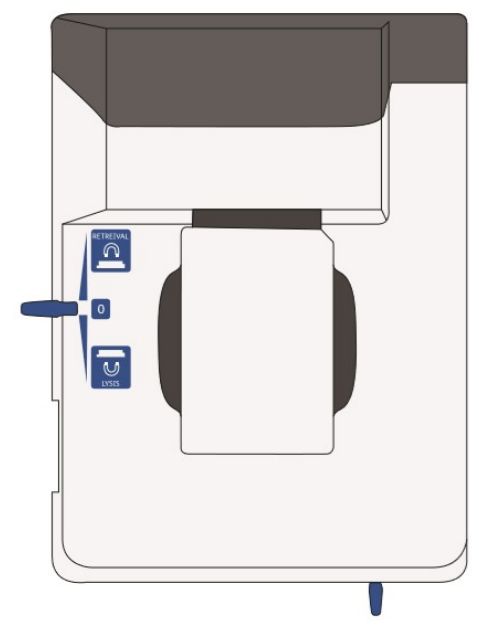

Figure 2. Top view of the Sample Loading Station showing slider positions for bead retrieval or lysis 
4. Move the front slider to OPEN (Figure 3).

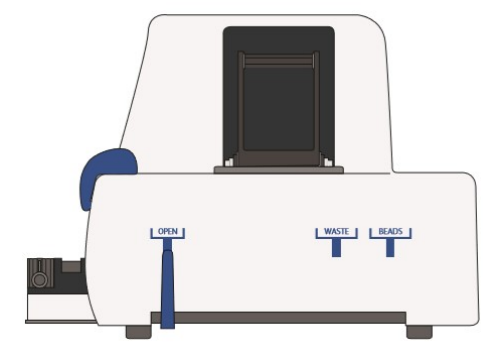

Figure 3. Front view of the Sample Loading Station showing the front slider to open the drawer

5. Remove the cap of a Waste Collection Container (PN 650000090), and insert both the container and a new $5 \mathrm{ml}$ LoBind Tube (Eppendorf) for bead retrieval into the appropriate slots in the drawer. Secure the cap of the $5 \mathrm{ml}$ LoBind Tube to the holder (Figure 4).

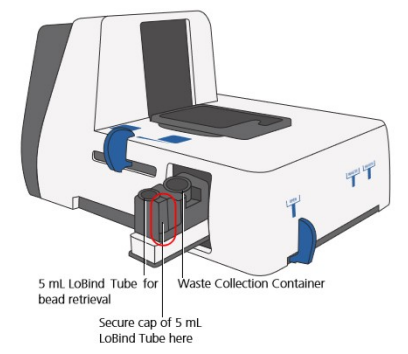

Figure 4. The drawer showing Waste Collection Container holder and the LoBind Tube holder for bead retrieval

6. Move the front slider to WASTE (Figure 5).

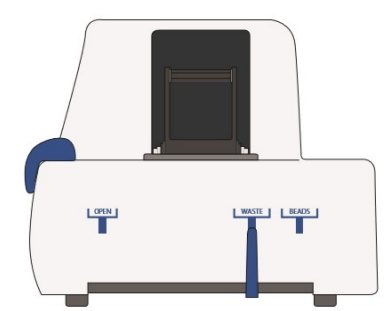

Figure 5. Front view of the Sample Loading Station showing the front slider to WASTE

7. Push the cartridge into the far end of the tray to match cartridge and tray notches. Lay the cartridge flat, and release it. Ensure that the cartridge is flat in the tray and the barcode faces out (Figure 6). 

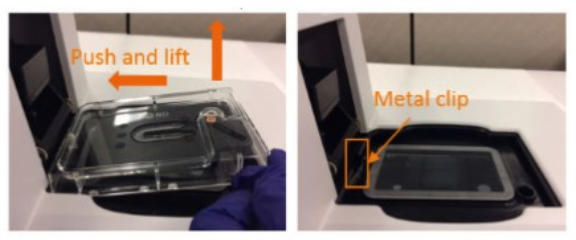

Figure 6. Top view into the Sample Loading Station showing cartridge holder

8. Load the cartridge with $700 \mu \mathrm{l}$ of $100 \%$ (absolute) ethyl alcohol using the $\mathrm{P} 1200 \mathrm{M}$ pipette in Prime/Treat mode.

9. Load the cartridge with $700 \mu \mathrm{l}$ of air using the $\mathrm{P} 1200 \mathrm{M}$ pipette in Prime/Treat mode.

10. Load the cartridge with $700 \mu \mathrm{l}$ of Cartridge Wash Buffer 1 (PN 650000060) with the P1200M pipette in Prime/Treat mode.

11. Leave the cartridge on the tray at room temperature for $1 \mathrm{~min}$.

B. Treating the surface of the cartridge

1. Load the cartridge with $700 \mu \mathrm{l}$ of air using the $\mathrm{P} 1200 \mathrm{M}$ pipette in Prime/Treat mode.

2. Load the cartridge with $700 \mu \mathrm{l}$ of Cartridge Wash Buffer 1 (PN 650000060) using the P1200M pipette in Prime/Treat mode.

3. Leave the cartridge on the tray at room temperature for $10 \mathrm{~min}$.

4. Load the cartridge with $700 \mu \mathrm{l}$ of air using the P1200M pipette in Prime/Treat mode.

5. Load the cartridge with $700 \mu \mathrm{l}$ of Cartridge Wash Buffer 2 (PN 650000061) using the P1200M pipette in Prime/Treat mode.

C. Staining cells with viability markers

1. Prepare a single cell suspension.

2. Add $3.1 \mu \mathrm{l}$ of $2 \mathrm{mM}$ Calcein $\mathrm{AM}$ and $3.1 \mu \mathrm{l}$ of $0.3 \mathrm{mM} \mathrm{DRAQ7}^{\mathrm{TM}}$ to the $620 \mu \mathrm{l}$ volume of cell suspension (1:200 dilution).

3. Gently pipet the suspension up and down to mix well.

4. Incubate the suspension in the dark in a water bath, incubator, or heat block at $37^{\circ} \mathrm{C}$ for $5 \mathrm{~min}$.

5. Pass the cells through a Falcon ${ }^{\circledR}$ Tube with Cell Strainer Cap.

6. Gently mix cells well by pipette, and then gently pipet $10 \mu \mathrm{l}$ of the cell suspension into one chamber of the INCYTO ${ }^{\text {TM }}$ disposable hemocytometer.

7. Insert the hemocytometer into the Hemocytometer Adapter so that the $A$ and $B$ sides of the hemocytometer align with $A$ and $B$ on the Hemocytometer Adapter (Figure 7). 


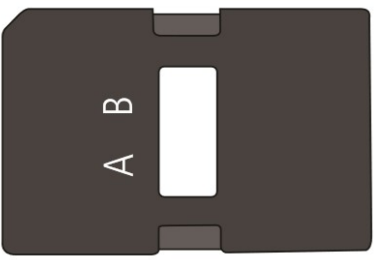

Figure 7. Top view of the Hemocytometer Adapter showing A and B sides of hemocytometer

8. Count the cells in the BD Rhapsody ${ }^{\top \mathrm{M}}$ Scanner $\leq 5$ min after loading.

D. Counting cells with the BD Rhapsody Scanner

1. Launch the BD Rhapsody Scanner software so that the main menu displays (Figure 8).

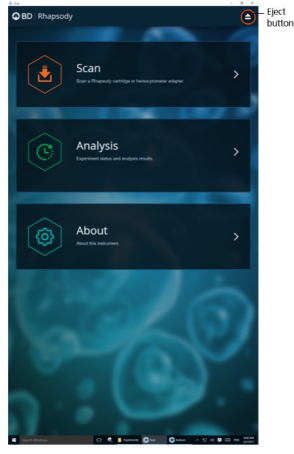

Figure 8. Interface of the BD Rhapsody Scanner software showing different options of Scan, Analysis, and About

2. Tap Scan. The tray door of the Scanner opens automatically, and the tray is ejected.

3. Place the Hemocytometer Adapter on the Scanner tray so that the notch of the adapter matches the corner notch, and the barcode faces toward the front of the instrument.

4. Tap Continue. The tray retracts, the door closes, and the Scanner displays the hemocytometer setup screen (Figure 9).

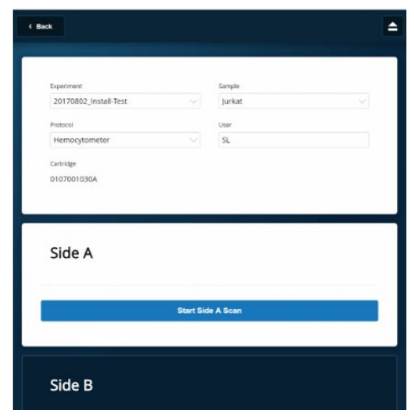

Figure 9. The hemocytometer setup screen, showing how to enter experiment name, sample name, protocol, and user 
5. Select the protocol from the drop-down menu. Enter the experiment name, sample name, protocol, and user.

6. Tap Side A or Side B, depending on which side the cells were loaded, and then Select Start Side A Scan or Start Side B Scan, as required.

7. After the scan is complete, tap OK.

8. View the total cell concentration and cell viability on the results screen. For example, cell concentrations and viabilities are displayed for each sample.

9. Tap Prepare at the top of the results screen to display the Samples Calculator screen.

10. Dispose of the hemocytometer according to local safety regulations.

11. Immediately proceed to Single cell capture on the BD Rhapsody ${ }^{\top \mathrm{M}}$ Cartridge.

E. Labeling samples with Sample Tags

1. Resuspend $1.2 \times 10^{4}$-/to $2 \times 10^{6}$ cells in $200 \mu$ of BD Stain Buffer (FBS).

2. Briefly centrifuge the Sample Tag tubes to collect the contents at the bottom.

3. For each sample, transfer $180 \mu \mathrm{l}$ of the cell suspension to a Sample Tag tube, and mix by pipette only.

4. Incubate the cell suspension at room temperature for $20 \mathrm{~min}$.

5. Add $200 \mu \mathrm{l}$ of BD Stain Buffer (FBS) to the cell suspension, and mix by pipette only.

6. Centrifuge the cells at $300 \times g$ for $5 \mathrm{~min}$.

7. Remove the supernatant without disturbing the pellet, and resuspend the pellet in $500 \mu \mathrm{l}$ of BD Stain Buffer (FBS). For low-abundance samples, leave $50 \mu \mathrm{l}$ of supernatant before resuspending the pellet in $500 \mu \mathrm{l}$ of BD Stain Buffer (FBS).

8. Centrifuge the cells at $300 \times g$ for $5 \mathrm{~min}$.

9. Remove the supernatant without disturbing the pellet, and resuspend the cells (stock cells) in $500 \mu \mathrm{l}$ of Sample Buffer from BD Rhapsody reagents.

10. Proceed immediately to the Staining cells with viability markers.

F. Loading cells in the BD Rhapsody ${ }^{\mathrm{TM}}$ Cartridge

1. According to the number of captured cells chosen, obtain the volume(s) of stock cell and volume of cold Sample Buffer (PN 650000062) to prepare a cell suspension of $\geq 650 \mu \mathrm{l}$ ( $\geq 610 \mu$ for low cell number samples) for loading into a BD Rhapsody Cartridge.

2. Load the cartridge on the tray with $700 \mu \mathrm{l}$ of air using the P1200M pipette in Prime/Treat mode.

3. Change the mode of the P1200M pipette to Cell Load.

4. With a manual pipette, gently pipet the cell suspension up and down to mix.

5. On the P1200M, press the pipette button once to aspirate $40 \mu \mathrm{l}$ of air, immerse the pipette tip in cell suspension, and then press the button again to aspirate $575 \mu \mathrm{l}$ of cold cell suspension.

6. Insert the tip of the pipette perpendicular to the port, seal the pipette tip against the gasket, and then press the button a third time to dispense $615 \mu$ of air and cells. 
7. On the Sample Loading Station: Leave the cartridge with loaded cells protected from light on the tray at room temperature for $15 \mathrm{~min}$. During incubation on the laboratory bench, prepare the Cell Capture Beads (PN 650000089) as indicated in the Procedure H.

G. Imaging cells in the cartridge

1. Navigate to the Scan application. The tray door of the Scanner opens automatically, and the tray is ejected.

2. Push the cartridge on the tray toward the back of the Sample Loading Station, and then pull up on the cartridge to remove it.

3. Place the cartridge on the Scanner tray so that the cartridge and tray notches match and the barcode faces toward the front of the instrument.

4. Tap Continue. The tray retracts, the door closes, and the Scanner displays the experiment setup screen.

5. Select from the drop-down menu or enter the experiment name, sample name, protocol, and user.

6. Tap Start Cell Load Scan. The tray retracts into the Scanner, and the door closes. If entered, the 15 min countdown time displays in the Cell Load window. The scan proceeds after the countdown completes. The scan takes an additional $\sim 4 \mathrm{~min}$ to complete.

7. After the scan is complete, tap Eject. The cartridge is ejected from the Scanner, and the remove cartridge screen is displayed.

H. Loading Cell Capture Beads and imaging

1. Push the cartridge into the far end of the BD Rhapsody Sample Loading Station tray to match cartridge and tray notches. Lay the cartridge flat and release it. Ensure that the cartridge is flat in the tray and the barcode faces out.

2. Change the mode of the BD Rhapsody P1200M pipette to Prime/Treat.

3. Load the cartridge with $700 \mu \mathrm{l}$ of air using the P1200M pipette in Prime/Treat mode.

4. Change the mode of the BD Rhapsody P1200M pipette to Bead Load.

5. Use a P1000 standard pipette to gently pipet the Cell Capture Beads (beads) in cold Sample Buffer (PN 650000062) up and down to mix, and, using the BD Rhapsody P1200M pipette in Bead Load mode, immediately load the cartridge with $630 \mu$ of beads.

6. Let the beads settle in the cartridge on the tray at room temperature for $3 \mathrm{~min}$.

7. Image the cartridge with the Scanner (Bead Load step).

8. Push the cartridge into the far end of the tray to match cartridge and tray notches. Lay the cartridge flat and release it. Ensure that the cartridge is flat in the tray and the barcode faces out.

9. Change the mode of the BD Rhapsody P1200M pipette to Wash.

10. Load the cartridge with $700 \mu \mathrm{l}$ of air using the BD Rhapsody P1200M pipette in Wash mode. 
11. Load the cartridge with $700 \mu$ of cold Sample Buffer (PN 650000062) using the BD Rhapsody $\mathrm{P} 1200 \mathrm{M}$ pipette in Wash mode.

12. Repeat Steps $\mathrm{H} 10-\mathrm{H} 11$ once for a total of two washes.

13. Image the cartridge with the BD Rhapsody Scanner.

I. Lysing cells and retrieving Cell Capture Beads

1. Add $75.0 \mu \mathrm{l}$ of $1 \mathrm{M} \mathrm{DTT}$ (PN 650000063) to one bottle of $15 \mathrm{ml}$ Lysis Buffer (PN 650000064), and then check the Add DTT box on the Lysis Buffer label.

2. Briefly vortex the lysis mix, and place it on ice.

3. Push the cartridge into the far end of the BD Rhapsody Sample Loading Station tray to match cartridge and tray notches. Lay the cartridge flat and release it. Ensure that the cartridge is flat in the tray and the barcode faces out.

4. Move the left slider to LYSIS. The (bottom) magnet is in the up position and is in contact with the cartridge.

5. Change the mode on the BD Rhapsody P1200M pipette to Lysis.

6. Load the cartridge with $550 \mu \mathrm{l}$ of Lysis Buffer with DTT using the BD Rhapsody P1200M pipette in Lysis mode.

7. Leave the cartridge on the tray at room temperature for $2 \mathrm{~min}$.

8. Ensure that a $5 \mathrm{ml}$ LoBind Tube (Eppendorf) was inserted into the drawer for bead retrieval.

9. Confirm that the mode on the BD ${ }^{\mathrm{TM}}$ Rhapsody P5000M pipette is Retrieval. The pipette is locked into this single mode.

10. Move the front slider to BEADS.

11. Move the left slider to RETRIEVAL. The (top) magnet is in the down position and is in contact with the cartridge.

12. Leave the Retrieval magnet in the down position for $30 \mathrm{~s}$.

13. Use the BD Rhapsody P5000M pipette to aspirate 5,000 $\mu \mathrm{l}$ of Lysis Buffer with DTT.

14. Press down on the BD Rhapsody P5000M pipette to seal the pipette tip against the gasket of the cartridge to avoid leaks.

15. Move the left slider to the middle (0) position, and immediately load the cartridge with $4,950 \mu \mathrm{l}$ of Lysis Buffer with DTT using the BD Rhapsody P5000M pipette. The Retrieval (top) magnet is in its full up position and is away from the cartridge.

16. Remove the pipette tip from the inlet valve of the cartridge before pressing the dial button once to purge the tip. Discard the pipette tip.

17. Move the front slider to OPEN, and then remove and cap the $5 \mathrm{ml}$ LoBind Tube.

18. Uncap the tube and place it on the large magnetic separation stand fitted with the $15 \mathrm{ml}$ tube adapter for $1 \mathrm{~min}$.

19. Image the cartridge with the BD Rhapsody Scanner (Retrieval step).

20. Proceed immediately to Performing reverse transcription and Exonuclease I treatment on the Cell Capture Beads to process the beads and begin reverse transcription. 
21. Appropriately dispose of the BD Rhapsody Cartridges according to biosafety level (BSL).

22. Appropriately dispose of the waste in the Waste Collection Container.

23. Appropriately dispose of the Lysis Buffer with DTT.

24. Wipe the Sample Loading Station with $10 \%$ bleach or $70 \%$ ethyl alcohol.

J. Performing reverse transcription on the Cell Capture Beads

1. After the $1 \mathrm{~min}$ incubation on the magnet and while leaving the $5 \mathrm{ml}$ LoBind Tube on the magnet, use a pipette to carefully remove all but $\sim 1 \mathrm{ml}$ of supernatant without disturbing the beads.

2. Remove the tube from the magnet, resuspend the $\sim 1 \mathrm{ml}$ beads by gently pipetting the suspension up and down, and then transfer the bead suspension to a new $1.5 \mathrm{ml}$ LoBind Tube.

3. If beads remain in the $5 \mathrm{ml}$ LoBind Tube, pipet an additional $0.5 \mathrm{ml}$ of Lysis Buffer with DTT into the tube, rinse the tube, and transfer the suspension to the $1.5 \mathrm{ml}$ LoBind Tube of beads.

4. Place the tube on the $1.5 \mathrm{ml}$ tube magnet for $\leq 2 \mathrm{~min}$, and then carefully remove and appropriately discard the supernatant without disturbing the beads and while leaving the tube on the magnet.

5. Remove the $1.5 \mathrm{ml}$ LoBind Tube from the magnet, and then pipet $1.0 \mathrm{ml}$ of cold Bead Wash Buffer (PN 650000065) to the tube. Gently mix the suspension by pipetting only. Do not vortex.

6. Place the tube on the $1.5 \mathrm{ml}$ tube magnet for $\leq 2 \mathrm{~min}$, and then carefully remove and appropriately discard the supernatant without disturbing the beads and while leaving the tube on the magnet.

7. Remove the $1.5 \mathrm{ml}$ LoBind Tube from the magnet, and then pipet $1.0 \mathrm{ml}$ of cold Bead Wash Buffer (PN 650000065) to the tube. Gently mix the suspension by pipetting only. Do not vortex.

8. Pipet the entire suspension to a new $1.5 \mathrm{ml}$ LoBind Tube, and put the tube on ice.

9. In the pre-amplification workspace, place a new $1.5 \mathrm{ml}$ LoBind Tube on ice, pipet the components in the following order to prepare the cDNA master mix (see Recipe 1).

10. Gently vortex and centrifuge the mix, and then put it back on ice.

11. Place the tube of washed beads on the $1.5 \mathrm{ml}$ tube magnet for $\leq 2 \mathrm{~min}$, and then carefully remove and appropriately discard the supernatant without disturbing the beads and while leaving the tube on the magnet.

12. Use a low retention tip to pipet $200 \mu \mathrm{l}$ of the cDNA mix to resuspend the beads. Gently mix the suspension by pipetting only. Do not vortex.

13. Transfer the bead suspension to a new $1.5 \mathrm{ml}$ LoBind Tube.

14. Ensure that the SmartBlock Thermoblock $1.5 \mathrm{ml}$ or equivalent is installed on the ThermoMixer.

15. Incubate the suspension on the ThermoMixer at $1,200 \mathrm{rpm}$ and $37^{\circ} \mathrm{C}$ for $20 \mathrm{~min}$.

16. After incubation, put the tube on ice. 
K. Treating the sample with Exonuclease I

1. In the pre-amplification workspace, prepare the Exonuclease I mix in a new $1.5 \mathrm{ml}$ LoBind Tube that is on ice by adding the components in the following order (Exonuclease I master mix) (see Recipe 2).

2. Gently vortex and centrifuge the mix, and then put it back on ice.

3. Place the tube of beads with cDNA mix on the $1.5 \mathrm{ml}$ tube magnet for $\leq 2 \mathrm{~min}$, and then carefully remove and appropriately discard the supernatant without disturbing the beads and while leaving the tube on the magnet.

4. Remove the tube from the magnet, and then use a low retention tip to pipet $200 \mu \mathrm{l}$ of Exonuclease I mix to the tube, Gently resuspend the beads by pipetting only. Do not vortex.

5. Incubate the suspension on the ThermoMixer at $1,200 \mathrm{rpm}$ and $37^{\circ} \mathrm{C}$ for $30 \mathrm{~min}$.

6. If the ThermoMixer or heat block needs to preheat to a different temperature $\left(80{ }^{\circ} \mathrm{C}\right.$ ThermoMixer or heat block), put the samples on ice until that temperature is reached.

7. Transfer the bead suspension with Exonuclease I to the ThermoMixer in the pre-amplification workspace at $80^{\circ} \mathrm{C}$ (no shaking) for $20 \mathrm{~min}$, or place the bead suspension in a heat block at $80^{\circ} \mathrm{C}$ for $20 \mathrm{~min}$.

8. Put the bead suspension on ice for $\sim 1 \mathrm{~min}$.

9. Place the tube on the $1.5 \mathrm{ml}$ tube magnet until the solution is clear ( $\leq 1 \mathrm{~min})$.

10. Carefully remove and appropriately discard the supernatant without disturbing the beads and while leaving the tube on the magnet.

11. Remove the tube from the magnet, and with a low retention tip, pipet $200 \mu \mathrm{l}$ of cold Bead Resuspension Buffer (PN 650000066) to gently resuspend the beads. Do not vortex.

L. Performing PCR1 on the Exonuclease I-treated sample

1. In the pre-amplification workspace, add these components in the following order to prepare the PCR1 reaction mix in a new $1.5 \mathrm{ml}$ LoBind Tube that is on ice with or without the PCR1 panel supplement (see Recipe 3).

2. Use reagents from the BD Rhapsody Targeted Amplification Kit (PN 633734). Gently vortex and centrifuge the mix, and place it back on ice until ready to use.

3. Place the tube of Exonuclease I-treated beads in cold Bead Resuspension Buffer (PN 650000066) from Step L1 or the subsample from Step L2 on the $1.5 \mathrm{ml}$ tube magnet. Carefully remove and appropriately discard the supernatant without disturbing the beads while leaving the tube on the magnet.

4. Remove the tube from the magnet, and then use a low retention tip to pipet $200 \mu \mathrm{l}$ of PCR 1 reaction mix to gently resuspend the beads.

5. Ensuring that the beads are fully suspended, pipet $50 \mu \mathrm{l}$ of the PCR1 reaction mix with beads into each of four $0.2 \mathrm{ml} \mathrm{PCR}$ tubes. Transfer any residual mix to one of the tubes.

6. Bring the PCR1 reaction mix into the post-amplification workspace. 
7. On the post-amplification thermal cycler, start the PCR1 thermal cycler program to ramp the heated lid and heat block of the thermal cycler to $>95^{\circ} \mathrm{C}$, and then pause the thermal cycler.

8. For each $0.2 \mathrm{ml} \mathrm{PCR}$ tube, gently mix the suspension of beads by pipetting only, and then immediately put the tube in the thermal cycler that has been ramped to $95^{\circ} \mathrm{C}$. Do not vortex.

9. Unpause Program 1 (see Recipe 4) on the PCR1 reaction mix with beads to run the thermal cycler program: Do not let the PCR1 reaction run overnight.

10. Briefly centrifuge the tubes to collect the contents at the bottom.

11. In the post-amplification workspace, resuspend the beads by pipetting, and combine the bead suspension from the four tubes into a new $1.5 \mathrm{ml}$ LoBind Tube. Use a small-volume pipette to transfer any residual suspension to the $1.5 \mathrm{ml}$ tube.

12. Place the tube of beads with PCR1 reaction on the $1.5 \mathrm{ml}$ tube magnet for $\leq 2 \mathrm{~min}$.

13. Carefully pipet the supernatant (PCR1 products) into a new $1.5 \mathrm{ml}$ LoBind Tube without disturbing the beads and while leaving the tube on the magnet.

14. Pipet 200 ul of cold Bead Resuspension Buffer (PN 650000066) to the beads. Gently resuspend the beads by pipetting only. Do not vortex.

15. Store the beads at $4{ }^{\circ} \mathrm{C}$ in the post-amplification workspace until all image analysis metrics pass.

M. Purifying the PCR1 product

1. In a new $5.0 \mathrm{ml}$ LoBind Tube, prepare $80 \%(\mathrm{v} / \mathrm{v})$ ethyl alcohol by pipetting $1,600 \mu \mathrm{l}$ of $100 \%$ (absolute) ethyl alcohol to $400.0 \mu \mathrm{l}$ of nuclease-free water. Cap and vortex the tube for $10 \mathrm{~s}$.

2. Vortex the AMPure XP beads at high speed for $1 \mathrm{~min}$. The beads should appear homogeneous and uniform in color.

3. Pipet $200 \mu$ l of AMPure XP beads (1X volume of amplification volume) to the tube containing the $200 \mu \mathrm{l}$ of supernatant (PCR1 products) from the amplification.

4. Vortex the suspension at high speed for $5 \mathrm{~s}$, and then briefly centrifuge the sample.

5. Keeping the tube on the magnet, gently pipet $500 \mu \mathrm{l}$ of $80 \%$ ethyl alcohol to the side of the tube opposite the pellet. Leave the tube open.

6. Purify the PCR1 products according to the following steps:

a. Pipet $200 \mu \mathrm{l}$ of AMPure XP beads (1 $\mathrm{x}$ volume of amplification volume) to the tube containing the $200 \mu \mathrm{l}$ of supernatant (PCR1 products) from the amplification.

b. Vortex the suspension at high speed for $5 \mathrm{~s}$, and then briefly centrifuge the sample.

c. Incubate the suspension at room temperature for $5 \mathrm{~min}$, and then place it on the $1.5 \mathrm{ml}$ tube magnet for $5 \mathrm{~min}$.

d. Carefully remove and appropriately discard only the supernatant without disturbing the AMPure XP beads while leaving the tube on the magnet.

e. Keeping the tube on the magnet, gently pipet $500 \mu \mathrm{l}$ of $80 \%$ ethyl alcohol to the side of the tube opposite the pellet. Leave the tube open.

f. Incubate the sample for $30 \mathrm{~s}$ on the magnet. 
g. Carefully remove and appropriately discard only the supernatant without disturbing the AMPure XP beads while leaving the tube on the magnet.

h. Repeat Steps M7-M9 once for a total of two washes.

i. Keeping the tube on the magnet, use a small-volume pipette to remove any residual supernatant from the tube.

j. Leave the tube open on the magnet to dry the AMPure XP beads at room temperature for $5 \mathrm{~min}$. Remove the tube from the magnet.

k. Pipet $30 \mu \mathrm{l}$ of Elution Buffer (PN 650000075) into the tube, and then repeatedly pipette the suspension up and down until the solution appears suspended, has no clumps, and is uniform in color.

I. Incubate the sample at room temperature for $2 \mathrm{~min}$, and then briefly centrifuge the tube to collect the contents at the bottom.

$\mathrm{m}$. Place the tube on the magnet until the solution is clear, usually $\leq 30 \mathrm{~s}$.

n. Pipet the entire eluate $(\sim 30 \mu \mathrm{l})$ into a new $1.5 \mathrm{ml}$ LoBind Tube. These are the purified PCR1 products.

N. Performing PCR2 on the PCR1 products

1. In the pre-amplification workspace, add these components in the following order to prepare the two reaction mixes, targeted PCR2 and Sample Tag PCR2, in separate, new $1.5 \mathrm{ml}$ LoBind Tubes that are on ice with or without the PCR2 panel supplement by using from the BD Rhapsody Targeted Amplification Kit (PN 633734) and the BD Single-Cell Multiplexing Kit Library Amplification Component (PN 633782) (see Recipes 5 and 6 for Targeted PCR2 reaction master mix and Sample Tag PCR2 reaction master mix).

2. Gently vortex and centrifuge the mixes, and place them back on ice until ready to use.

3. Bring the PCR2 reaction mixes to the post-amplification workspace.

4. Use two separate and new $0.2 \mathrm{ml} \mathrm{PCR}$ tubes for:

a. Targeted PCR: Pipet $5.0 \mu \mathrm{l}$ of the purified PCR1 products to $45 \mu \mathrm{l}$ of the targeted PCR2 reaction, and mix by pipetting for a total of $50 \mu$.

b. Sample Tag PCR: Pipet $5.0 \mu \mathrm{l}$ of the purified PCR1 products to $45 \mu \mathrm{l}$ of the Sample Tag PCR2 reaction, and mix by pipetting for a total of $50 \mu$.

5. Gently vortex and centrifuge the tubes.

6. Run Program 1 (see Recipe 4) on the PCR2 master mix in the post-amplification thermal cycler.

O. Purifying the PCR2 products

1. Vortex the AMPure XP beads at high speed for $1 \mathrm{~min}$. The beads should appear homogeneous and uniform in color.

2. Briefly centrifuge the tube of PCR2 products to collect contents at the bottom.

3. Pipet AMPure XP beads according to the specified ratio (volume) into each tube containing $50 \mu \mathrm{l}$ products. 
a. Targeted PCR2 products: Use $0.7 \times(35 \mu \mathrm{l})$ of Agencourt AMPure XP magnetic beads.

b. Sample Tag PCR2 products: Use $1 \times(50 \mu \mathrm{l})$ of Agencourt AMPure XP magnetic beads.

4. Vortex the suspensions at high speed for $5 \mathrm{~s}$, and then briefly centrifuge the sample $(<1 \mathrm{~s})$.

5. Incubate the suspensions at room temperature for $5 \mathrm{~min}$, and then place them on the strip tube magnet for 3 min.

6. Carefully remove and appropriately discard only the supernatant without disturbing the AMPure $\mathrm{XP}$ beads while leaving the tubes on the magnet.

7. Keeping the tubes on the magnet, gently pipet $200 \mu \mathrm{l}$ of fresh $80 \%$ ethyl alcohol to the side of each tube opposite the pellet. Leave the tubes open.

8. Incubate the samples for $30 \mathrm{~s}$ on the magnet.

9. Carefully remove and appropriately discard only the supernatant without disturbing the AMPure XP beads while leaving the tubes on the magnet.

10. Repeat Steps 07-09 once for a total of two washes.

11. Keeping the tubes on the magnet, use a small-volume pipette to remove any residual supernatant from the tubes.

12. Leave the tubes open on the magnet to dry the AMPure XP beads at room temperature for $3 \mathrm{~min}$. Remove the tubes from the magnet.

13. Pipet $30 \mu \mathrm{l}$ of Elution Buffer (PN 650000075) into each tube, close the tubes, and then pulse vortex to completely resuspend the AMPure XP beads.

14. Briefly centrifuge the tubes to collect the contents at the bottom, and then incubate the samples at room temperature for $2 \mathrm{~min}$.

15. Place the tubes on the strip tube magnet until the solution is clear, usually $\leq 30 \mathrm{~s}$.

16. For each tube, pipet the entire eluate $(\sim 30 \mu \mathrm{l})$ into a new $1.5 \mathrm{ml}$ LoBind Tube. These two tubes are the purified targeted and Sample Tag PCR2 products.

P. Quantifying the PCR2 products and Performing final amplification of pooled samples

1. Estimate the concentration by quantifying $2 \mu \mathrm{l}$ of the targeted and Sample Tag PCR2 products with a Qubit ${ }^{\mathrm{TM}}$ Fluorometer using the Qubit ${ }^{\mathrm{TM}}$ dsDNA HS Assay Kit as per the manufacturer's instructions.

2. Dilute an aliquot of the PCR2 products to the following concentrations in Elution Buffer (PN 650000075):

a. Targeted PCR2 products: $10 \mathrm{ng} / \mu \mathrm{l}$.

b. Sample Tag products: $0.5 \mathrm{ng} / \mu \mathrm{l}$.

3. In the pre-amplification workspace, prepare the 1 library $+20 \%$ overage of the final amplification mix for each of the two products. Add these components in the following order to prepare the mix in a new $1.5 \mathrm{ml}$ LoBind Tube that are on ice (see Recipes 7 and 8) by using the BD Rhapsody Targeted Amplification Kit (PN 633734) and BD Single-Cell Multiplexing Kit Library Amplification Component (PN 633782).

4. Gently vortex and centrifuge the mixes, and place them back on ice until ready to use. 
5. Bring the final amplification into the post-amplification workspace.

6. Use two separate and new $0.2 \mathrm{ml}$ PCR tubes for:

a. Targeted library master mix: Pipet $3.0 \mu \mathrm{l}$ of $10 \mathrm{ng} / \mu \mathrm{l}$ of targeted PCR2 products to $47.0 \mu \mathrm{l}$ of the targeted final amplification mix for a total of $50 \mu \mathrm{l}$. Gently vortex and centrifuge the mix.

b. Sample Tag library master mix: Pipet $3.0 \mu \mathrm{l}$ of $0.5 \mathrm{ng} / \mu \mathrm{l}$ of Sample Tag PCR2 products to $47.0 \mu \mathrm{l}$ of the Sample Tag final amplification mix for a total of $50 \mu \mathrm{l}$. Gently vortex and centrifuge the mix.

7. Run Program 2 on the final amplification master mix in the post-amplification thermal cycler to prepare the final amplification products (see Recipe 9).

8. Safe stopping point: samples can be left for up to $1 \mathrm{~h}$ in the thermal cycler at $4{ }^{\circ} \mathrm{C}$ or freezer at $-20^{\circ} \mathrm{C}$ for a few days.

Q. Purifying the final amplification products

1. Vortex the AMPure XP beads at high speed for $1 \mathrm{~min}$. The beads should appear homogeneous and uniform in color.

2. Briefly centrifuge the tubes of final amplification products to collect the contents at the bottom.

3. Pipet AMPure XP beads according to the specified ratio (volume) into each tube containing $50 \mu \mathrm{l}$ products:

a. Final targeted library: Use $0.6 \mathrm{x}$ (30 $\mu \mathrm{l})$ of Agencourt AMPure XP magnetic beads.

b. Final Sample Tag library: Use 1x (50 $\mu$ l) of Agencourt AMPure XP magnetic beads.

4. Vortex the suspensions at high speed for $5 \mathrm{~s}$, and then briefly centrifuge the sample.

5. Incubate the suspensions at room temperature for $5 \mathrm{~min}$, and then place them on the strip tube magnet for $3 \mathrm{~min}$.

6. Carefully remove and appropriately discard only the clear liquid supernatant without disturbing the AMPure XP beads and while leaving the tube on the magnet.

7. Keeping the tube on the magnet, pipet $200 \mu$ of fresh $80 \%$ ethyl alcohol to the side of each tube opposite the pellet. You can leave the tube open.

8. Incubate the samples for $30 \mathrm{~s}$ on the magnet.

9. Carefully remove and appropriately discard the supernatant without disturbing the AMPure XP beads and while leaving the tubes on the magnet.

10. Repeat Steps Q7-Q9 once for a total of two washes.

11. Keeping the tubes on the magnet, use a small-volume pipette to remove any residual supernatant from the tube.

12. Leave the tube open on the magnet to dry the AMPure XP beads at room temperature for $3 \mathrm{~min}$. Remove the tubes from the magnet.

13. Pipet $30 \mu \mathrm{l}$ of Elution Buffer (PN 650000075) into the tubes, close the tubes, and then pulse vortex to completely resuspend the AMPure XP beads.

14. Briefly centrifuge the tubes to collect the contents at the bottom, and then incubate the samples at room temperature for $2 \mathrm{~min}$. 
15. Place the tubes on the magnet until the solution is clear, usually $\leq 30 \mathrm{~s}$.

16. For each tube, pipet the entire eluate $(\sim 30 \mu \mathrm{l})$ into a new $1.5 \mathrm{ml}$ LoBind Tube. These two tubes are the final sequencing libraries.

R. Performing quality control on the final sequencing libraries

1. Estimate the concentration by quantifying $2 \mu \mathrm{l}$ of the final amplified libraries with a Qubit Fluorometer using the Qubit ${ }^{\mathrm{TM}}$ dsDNA HS Kit as per the manufacturer's instructions. The expected library concentration is $>1.5 \mathrm{ng} / \mu \mathrm{l}$. If it is necessary to dilute the library, dilute it in Elution Buffer (PN 650000075).

2. Set the size range to $200-2,000 \mathrm{bp}$. Measure the size ranges of the libraries using the Agilent Bioanalyzer with the High Sensitivity Kit (Agilent PN 5067-4626) for 50-7,000 bp, 5-1,000 pg/ $\mathrm{\mu l}$. The Sample Tag library shows a fragment distribution of 200-350 bp. The BD Rhapsody targeted library shows a fragment distribution that depends on the panel used.

\section{S. Sequencing}

1. Minimum of $75 \times 75$ paired read length with $\sim 20 \%$ of the PHIX control is required.

2. Sequencing depth is dependent on application. For cell type clustering, shallow sequencing is sufficient. For in-depth analysis, such as comparison across multiple libraries, deep sequencing is recommended. BD Biosciences recommends meeting the requirement for recursive substitution error correction (RSEC) sequencing depth of $\geq 6$ in order to reach the threshold of sequencing saturation where most molecules of the library have been recovered. RSEC sequencing depth is reported by the analysis pipeline. The actual sequencing reads/cell required to achieve this depth can vary, because it depends on the chosen gene panel, number of cells, and sequencing run quality.

3. For the Sample Tag library, pool samples of the same type: $\geq 120$ reads/cell.

4. Illumina HiSeq 4000 is used for the sequencing.

\section{Data analysis}

1. FASTQ files were generated using the Illumina bcl2fastq pipeline for data extraction.

2. The single cell genomics data analysis is performed on the Seven Bridges Genomics platform using the Rhapsody Analysis Pipeline to generate molecular counts per cell, read counts per cell, metrics, and an alignment file.

3. Clustering analysis is based on single-cell gene expression profiles.

4. Further data analysis was done by using MATLAB and BD Data View.

Example data: We stained cells with 18 DNA-labeled antibodies and loaded to the BD cartridges on the sample loading instrument then used barcoded beads to capture the cells that identify the original cell types, then analyzed those linked barcodes using the Illumina Hiseq4000 sequencer. 
We prepared three types of libraries to identify Sample Tag, AbSeq, and mRNA seq respectively and the final sequencing pool for each set of samples was mixed by percentage of $5 \%, 75 \%$, and $20 \%$ in molarity. The overall sequencing depth for all metrics met all criteria of the BD Rhapsody recommendations (Table 1). The FASTQ files generated on Hiseq4000 were uploaded into the Seven Bridge Genomics, and the BD Rhapsody single cell analysis pipeline was used for the AbSeq. Three pooled libraries were prepared accordingly from 10 samples and sequenced. The sample tag libraries showed even distributions of individual sample in each pool (Figure 10).

Table 1. Metrics of the sequences of Sample Tag, AbSeq, and mRNA libraries

\begin{tabular}{|c|c|c|c|}
\hline & Pool1 & Pool2 & Pool3 \\
\hline Total reads & $356.5 \mathrm{M}$ & $347.7 \mathrm{M}$ & $364 \mathrm{M}$ \\
\hline Q30 & $66.2 \%$ & $64.99 \%$ & $66.65 \%$ \\
\hline$\%$ reads assigned to cell labels & $91.9 \%$ & $91.8 \%$ & $90 \%$ \\
\hline$\%$ reads aligned to amplicons & $87.9 \%$ & $87.5 \%$ & $85.6 \%$ \\
\hline Aligned reads (mRNA) / per cell (avg RSEC) & $81 \mathrm{M} / 2853$ & $29.3 \mathrm{M} / 847.2$ & $115.6 \mathrm{M} / 6396.4$ \\
\hline Aligned reads (AbSeq) / per cell (avg RSEC) & $215 M / 2903$ & $248 \mathrm{M} / 4675$ & $161.8 \mathrm{M} / 4731.1$ \\
\hline Aligned reads (ST) / per cell (avg) & $17.6 \mathrm{M} / 1713$ & $15.8 \mathrm{M} / 1314$ & $18.3 \mathrm{M} / 3584$ \\
\hline \# of cells detected & 10298 & 12005 & 5103 \\
\hline \# mRNA targets detected & $379 / 399$ & $365 / 399$ & $357 / 399$ \\
\hline \# of AbSeq targets detected & $18 / 18$ & $18 / 18$ & $18 / 18$ \\
\hline$\%$ reads from putative cells (RSEC) mRNA & $38.4 \%$ & $37.5 \%$ & $30.4 \%$ \\
\hline$\%$ reads from putative cells (RSEC) AbSeq & $13.85 \%$ & $22.6 \%$ & $14.9 \%$ \\
\hline mRNA Seq saturation & $96 \%$ & $93.5 \%$ & $96.9 \%$ \\
\hline AbSeq Seq saturation & $27.6 \%$ & $34.7 \%$ & $40.3 \%$ \\
\hline mRNA Mean mols per cell (RSEC) & 160 & 108.8 & 213.2 \\
\hline AbSeq Mean mols per cell (RSEC) & 2301 & 3693.6 & 3555.4 \\
\hline mRNA Mean targets per cell (RSEC) & 65.6 & 45.4 & 70.2 \\
\hline AbSeq Mean targets per cell (RSEC) & 17.98 & 17.98 & 17.8 \\
\hline
\end{tabular}



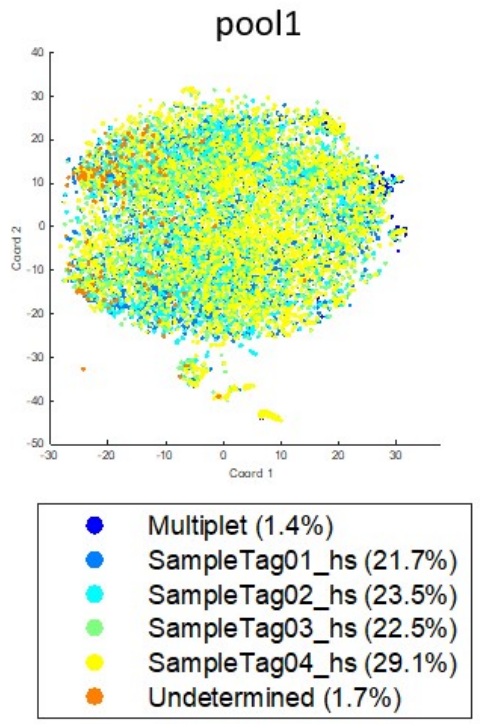
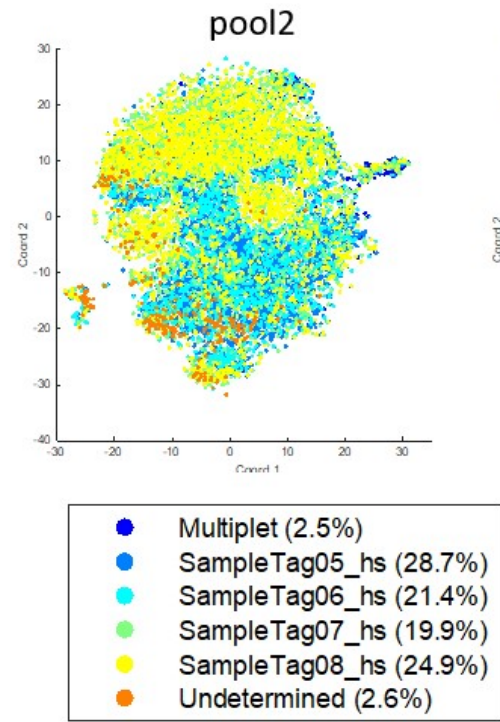
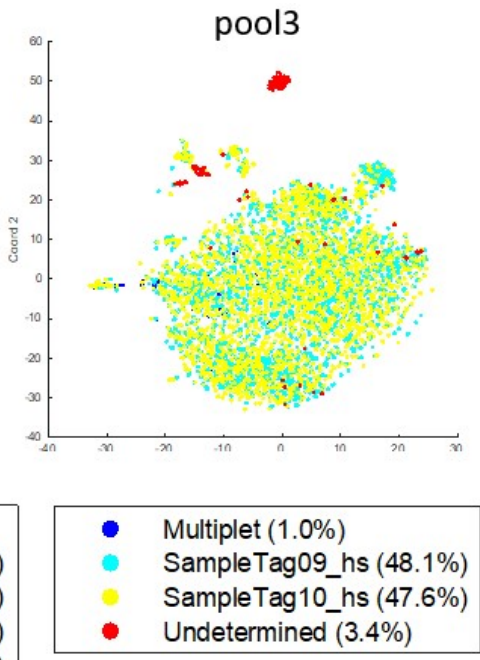

Figure 10. Sample tag calling indicated each sample had relatively even distributions and the sequencing depth met minimal requirement for enough sequencing coverage

\section{Notes}

1. If using biological samples that contain red blood cell contamination, red blood cell lysis is required. Use a standard erythrocyte lysis protocol and then proceed with cell staining.

2. If the cells are not resuspended in cold Sample Buffer (PN 650000062), centrifuge the cell suspension at $300 \times \mathrm{g}$ for $5 \mathrm{~min}$, aspirate the supernatant, and leave $\sim 20 \mu \mathrm{l}$ of the residual supernatant. Add up to $620 \mu \mathrm{l}$ total volume of cold Sample Buffer, and then proceed with cell staining.

3. Make fresh $80 \%$ ethyl alcohol, and use it in $\leq 24 \mathrm{~h}$.

4. Stopping point: The purified PCR2 products can be stored at $4{ }^{\circ} \mathrm{C}$ before proceeding on the same day or at $-20^{\circ} \mathrm{C}$ for $\leq 6$ months. 


\section{Recipes}

1. cDNA master mix prep

\begin{tabular}{|l|l|l|l|}
\hline Component & $\begin{array}{l}\text { 1 library } \\
(\mu \mathrm{l})\end{array}$ & $\begin{array}{l}\text { 1 library }+20 \% \\
\text { overage }(\mu \mathrm{l})\end{array}$ & $\begin{array}{l}\text { 2 libraries + 10\% } \\
\text { overage }(\mu \mathrm{l})\end{array}$ \\
\hline Nuclease-Free Water (PN 650000076) & 106.7 & 128.0 & 235.0 \\
\hline RT Buffer (PN 650000067) & 40.0 & 48.0 & 88.0 \\
\hline dNTPs (PN 650000077) & 20.0 & 24.0 & 44.0 \\
\hline RT 0.1 M DTT (PN 650000068) & 10.0 & 12.0 & 22.0 \\
\hline RT/PCR Enhancer (PN 650000070) & 3.3 & 4.0 & 7.3 \\
\hline RNase Inhibitor (PN 650000078) & 10.0 & 12.0 & 22.0 \\
\hline Reverse Transcriptase (PN 650000069) & 10.0 & 12.0 & 22.0 \\
\hline Total & 200.0 & 240.0 & 440.0 \\
\hline
\end{tabular}

2. Exonuclease I master mix

\begin{tabular}{|l|l|l|l|}
\hline Component & $\begin{array}{l}1 \text { library } \\
(\mu \mathrm{l})\end{array}$ & $\begin{array}{l}\text { l library }+20 \% \\
\text { overage }(\mu \mathrm{l})\end{array}$ & $\begin{array}{l}2 \text { libraries }+10 \% \\
\text { overage }(\mu \mathrm{l})\end{array}$ \\
\hline Nuclease-Free Water (PN 650000076) & 170.0 & 204.0 & 374.0 \\
\hline 10x Exonuclease I Buffer (PN 650000071) & 20.0 & 24.0 & 44.0 \\
\hline Exonuclease I (PN 650000072) & 10.0 & 12.0 & 22.0 \\
\hline Total & 200.0 & 240.0 & 440.0 \\
\hline
\end{tabular}

3. PCR1 reaction master mix

\begin{tabular}{|l|l|l|l|}
\hline Component & $\begin{array}{l}1 \text { library } \\
(\mu \mathrm{l})\end{array}$ & $\begin{array}{l}\text { 1 library }+20 \% \\
\text { overage }(\mu \mathrm{l})\end{array}$ & $\begin{array}{l}2 \text { libraries }+10 \% \\
\text { overage }(\mu \mathrm{l})\end{array}$ \\
\hline Nuclease-Free Water (PN 650000076) & Up to 30.0 & Up to 36.0 & Up to 66.0 \\
\hline PCR MasterMix (PN 650000073) & 100.0 & 120.0 & 220.0 \\
\hline Universal Oligo (PN 650000074) & 20.0 & 24.0 & 44.0 \\
\hline RT/PCR Enhancer (PN 650000070) & 10.0 & 12.0 & 22.0 \\
\hline PCR1 primer panel & 40.0 & 48.0 & 88.0 \\
\hline Total & 200.0 & 240.0 & 440.0 \\
\hline
\end{tabular}


4. PCR Program 1

\begin{tabular}{|l|l|l|l|}
\hline Step & Cycles & Temperature & Time \\
\hline Hot start & 1 & $95^{\circ} \mathrm{C}$ & $3 \mathrm{~min}$ \\
\hline Denaturation & 15 & $95^{\circ} \mathrm{C}$ & $30 \mathrm{~s}$ \\
\hline Annealing & 15 & $60^{\circ} \mathrm{C}$ & $3 \mathrm{~min}$ \\
\hline Extension & 15 & $72^{\circ} \mathrm{C}$ & $1 \mathrm{~min}$ \\
\hline Final extension & 1 & $72^{\circ} \mathrm{C}$ & $5 \mathrm{~min}$ \\
\hline Hold & 1 & $4{ }^{\circ} \mathrm{C}$ & $\infty$ \\
\hline
\end{tabular}

5. Targeted PCR2 reaction master mix

\begin{tabular}{|l|l|l|l|}
\hline Component & $\begin{array}{l}1 \text { library } \\
(\mu \mathrm{l})\end{array}$ & $\begin{array}{l}\text { 1 library + 20\% } \\
\text { overage }(\mu \mathrm{l})\end{array}$ & $\begin{array}{l}\text { 2 libraries + 10\% } \\
\text { overage }(\mu \mathrm{l})\end{array}$ \\
\hline Nuclease-Free Water (PN 650000076) & Up to 8.0 & Up to 9.6 & Up to 17.6 \\
\hline PCR MasterMix (PN 650000073) & 25.0 & 30.0 & 55.0 \\
\hline Universal Oligo (PN 650000074) & 2.0 & 2.4 & 4.4 \\
\hline PCR2 primer panel & 10.0 & 12.0 & 22.0 \\
\hline Total & 45.0 & 54.0 & 99.0 \\
\hline
\end{tabular}

6. Sample Tag PCR2 reaction master mix

\begin{tabular}{|l|l|l|}
\hline Component & 1 library $(\mu \mathrm{l})$ & 1 library $+20 \%$ overage $(\mu \mathrm{l})$ \\
\hline Nuclease-Free Water (PN 650000076) & 15.0 & 18.0 \\
\hline PCR MasterMix (PN 91-1052) & 25.0 & 30.0 \\
\hline Universal Oligo (PN 91-1054) & 2.0 & 2.4 \\
\hline Sample Tag PCR2 Primer (91-1061) & 3.0 & 3.6 \\
\hline Total & 45.0 & 54.0 \\
\hline
\end{tabular}

7. Targeted final amplification master mix

\begin{tabular}{|l|l|l|}
\hline Component & 1 library $(\mu \mathrm{l})$ & 1 library + 20\% overage $(\mu \mathrm{l})$ \\
\hline Nuclease-Free Water (PN 650000076) & 18.0 & 21.6 \\
\hline PCR MasterMix (PN 650000073) & 25.0 & 30.0 \\
\hline Library Forward Primer (PN 650000079) & 2.0 & 2.4 \\
\hline $\begin{array}{l}\text { Library Reverse Primer 1-4 } \\
\text { (PN 650000080, 650000091-93) }\end{array}$ & 2.0 & 2.4 \\
\hline Total & 47.0 & 56.4 \\
\hline
\end{tabular}


8. Sample Tag final amplification master mix

\begin{tabular}{|l|l|l|}
\hline Component & 1 library $(\mu \mathrm{l})$ & 1 library $+20 \%$ overage $(\mu \mathrm{l})$ \\
\hline Nuclease-Free Water (PN 650000076) & 18.0 & 21.6 \\
\hline PCR MasterMix (PN 91-1052) & 25.0 & 30.0 \\
\hline Library Forward Primer (PN 91-1055) & 2.0 & 2.4 \\
\hline $\begin{array}{l}\text { Library Reverse Primer 1-4 } \\
\text { (PN 650000080, 650000091-93) }\end{array}$ & 2.0 & 2.4 \\
\hline Total & 47.0 & 56.4 \\
\hline
\end{tabular}

9. PCR Program 2

\begin{tabular}{|l|l|l|l|}
\hline Step & Cycles & Temperature & Time \\
\hline Hot start & 1 & $95^{\circ} \mathrm{C}$ & $5 \mathrm{~min}$ \\
\hline Denaturation & 8 & $98^{\circ} \mathrm{C}$ & $15 \mathrm{~s}$ \\
\hline Annealing & 8 & $60^{\circ} \mathrm{C}$ & $30 \mathrm{~s}$ \\
\hline Extension & 8 & $72^{\circ} \mathrm{C}$ & $30 \mathrm{~s}$ \\
\hline Final extension & 1 & $72^{\circ} \mathrm{C}$ & $1 \mathrm{~min}$ \\
\hline Hold & 1 & $4{ }^{\circ} \mathrm{C}$ & $\infty$ \\
\hline
\end{tabular}

\section{Acknowledgments}

This work was supported by NIH grants 1U19AI104209 and 5U19Al110491. We thank Mike Snyder's lab (SCMGM) for sequencing, and Jessica Duller, Christina Chang \& Jonathan Slasinski at BD Genomics for helpful discussions and technical support. The authors declare no conflict of interest.

\section{References}

1. BDgenomics. (2018). Single Cell Targeted Library Preparation with the BD Rhapsody ${ }^{\top M}$ SingleCell Analysis System User Guide, Doc ID: 47395 Rev. 3.0 01/2018.

2. Fan, H. C., Fu, G. K. and Fodor, S. P. (2015). Expression profiling. Combinatorial labeling of single cells for gene expression cytometry. Science 347(6222): 1258367.

3. Fu, G. K., Wilhelmy, J., Stern, D., Fan, H. C. and Fodor, S. P. (2014a). Digital encoding of cellular mRNAs enabling precise and absolute gene expression measurement by singlemolecule counting. Anal Chem 86(6): 2867-2870.

4. Fu, G. K., Xu, W., Wilhelmy, J., Mindrinos, M. N., Davis, R. W., Xiao, W. and Fodor, S. P. (2014b). Molecular indexing enables quantitative targeted RNA sequencing and reveals poor efficiencies in standard library preparations. Proc Natl Acad Sci USA 111(5): 1891-1896.

5. Han, A., Glanville, J., Hansmann, L. and Davis, M. M. (2014). Linking T-cell receptor sequence to functional phenotype at the single-cell level. Nat Biotechnol 32(7): 684-692. 
6. Lam, R., Shum, E., Chang, C. C., Lam, G., Bansal, N., Shah, H., Jensen, D., Hu, J., Ghadiali, J., Martin, J., Rosenfeld, D. and Fan, H. C. (2018). Dissection of single-cell gene regulation by simultaneous digital mRNA and protein quantification. The Journal of Immunology 200(1 Supplement): 120.141-120.141.

7. Landhuis, E. (2018). Single-cell approaches to immune profiling. Nature 557(7706): 595-597.

8. Martin, J., Fan, H. C. and Shum, E. (2018). Oligo-conjugated antibodies (Ab-seq) and massively parallel single cell sequencing reveal the high parameter correlation of protein and mRNA expression in individual immune cells. J Immunol 200 (1 Supplement): 120.133-120.133.

9. Newell, E. W., Sigal, N., Bendall, S. C., Nolan, G. P. and Davis, M. M. (2012). Cytometry by time-of-flight shows combinatorial cytokine expression and virus-specific cell niches within a continuum of $\mathrm{CD}^{+} \mathrm{T}$ cell phenotypes. Immunity 36(1): 142-152.

10. Shapiro, E., Biezuner, T. and Linnarsson, S. (2013). Single-cell sequencing-based technologies will revolutionize whole-organism science. Nat Rev Genet 14(9): 618-630.

11. Wills, Q. F., Livak, K. J., Tipping, A. J., Enver, T., Goldson, A. J., Sexton, D. W. and Holmes, C. (2013). Single-cell gene expression analysis reveals genetic associations masked in wholetissue experiments. Nat Biotechnol 31(8): 748-752. 